\title{
A Korovkin Type Approximation Theorem and Its Applications
}

\author{
Malik Saad Al-Muhja \\ Department of Mathematics and Computer Application, College of Sciences, University of Al-Muthanna, Samawa, Iraq
}

Correspondence should be addressed to Malik Saad Al-Muhja; dr.al-muhja@hotmail.com

Received 28 January 2014; Accepted 20 March 2014; Published 17 April 2014

Academic Editor: Ivanka Stamova

Copyright (C) 2014 Malik Saad Al-Muhja. This is an open access article distributed under the Creative Commons Attribution License, which permits unrestricted use, distribution, and reproduction in any medium, provided the original work is properly cited.

We present a Korovkin type approximation theorem for a sequence of positive linear operators defined on the space of all real valued continuous and periodic functions via $A$-statistical approximation, for the rate of the third order Ditzian-Totik modulus of smoothness. Finally, we obtain an interleave between Riesz's representation theory and Lebesgue-Stieltjes integral-i, for Riesz's functional supremum formula via statistical limit.

\section{Introduction and Main Results}

Some will accept the notes and definitions used in this paper. The concept of $A$-statistical approximation for regular summability matrix (see $[1,2]$ ). Let $A=\left(a_{n k}\right), n, k=1,2, \ldots$, be an infinite summability matrix. For a given sequence $x=$ $\left(x_{k}\right)$, the $A$-transform of $x$, denoted by $A x=(A x)_{n}$, is given by $(A x)_{n}=\sum_{k=1}^{\infty} a_{n k} x_{k}$, provided that the series converges for each $n$. $A$ is said to be regular if $\lim _{n \rightarrow \infty}(A x)_{n}=L$, whenever $\lim x=L$. Then $\lim _{n \rightarrow \infty} a_{n k}=0$, for all $k \in N$. In [3], Dzyubenko and Gilewicz have given the notion.

$A$ is nonnegative regular summability matrix. Then $x$ is $A$-statistically convergent to $L$, if, for every $\epsilon>0$, $\lim _{n \rightarrow \infty} \sum_{k:\left|x_{k}-L\right| \geq \epsilon} a_{n k}=0$

We denote by $\mathrm{C}_{2 \pi}(\mathscr{R})$ the space of all $2 \pi$-periodic and continuous functions on $\mathscr{R}$. Endowed with the norm $\|\cdot\|_{2 \pi}$, this space is a Banach space, where $\|f\|_{2 \pi}=\sup \{|f(t)|$ : $\left.f \in \mathrm{C}_{2 \pi}(\mathscr{R}), t \in \mathscr{R}\right\}$. Now, recall that, in [4], the $m$ th order Ditzian-Totik modulus of smoothness in the uniform metric is given by

$$
\omega_{m}^{\phi}(f, \delta,[a, b])=\sup _{0<h \leq \delta}\left\|\Delta_{h \phi(x)}^{m}(f, x,[a, b])\right\|_{[a, b]},
$$

where

$$
\begin{aligned}
& \Delta_{h}^{m}(f, x,[a, b]) \\
& = \begin{cases}\sum_{i=0}^{m}\left(\begin{array}{c}
m \\
i
\end{array}\right)(-1)^{m-i} \\
\times f\left(x-\frac{m h}{2}+i h\right), & \text { if } x \pm \frac{m h}{2} \in[a, b] \\
0, & \text { o.w }\end{cases}
\end{aligned}
$$

is the symmetric $m$ th difference. We have to recall the Korovkin type theorem.

Theorem 1 (see [2]). Let $A=\left(A^{n}\right)_{n \geq 1}$ be a sequence of infinite nonnegative real matrices such that $\sup _{n, k} \sum_{j=1}^{\infty} a_{k j}^{n}<\infty$ and let $\left\{L_{j}\right\}$ be a sequence of positive linear operators mapping $\mathrm{C}_{2 \pi}(\mathscr{R})$ into $\mathrm{C}_{2 \pi}(\mathscr{R})$. Then, for all $f \in \mathrm{C}_{2 \pi}(\mathscr{R})$, we have $\lim _{k \rightarrow \infty} \sum_{j=1}^{\infty} a_{k j}^{n}\left\|L_{j} f-f\right\|_{2 \pi}=0$ uniformly in $n$, if and only if $\lim _{k \rightarrow \infty} \sum_{j=1}^{\infty} a_{k j}^{n}\left\|L_{j} f_{i}-f_{i}\right\|_{2 \pi}=0(i=1,2,3)$, uniformly in $n$, where $f_{1}(t)=1, f_{2}(t)=\cos t$, and $f_{3}(t)=\sin t$, for all $t \in \mathscr{R}$.

It is worth noting that the statistical analog of Theorem 1 has been studied by Radu [2], as follows. 
Theorem 2. Let $A=\left(A^{n}\right)_{n \in N}$ be a sequence of nonnegative regular summability matrices and let $\left\{L_{j}\right\}$ be a sequence of positive linear operators mapping $\mathrm{C}_{2 \pi}(\mathscr{R})$ into $\mathrm{C}_{2 \pi}(\mathscr{R})$. Then, for all $f \in \mathrm{C}_{2 \pi}(\mathscr{R})$, we have st ${ }_{A}-\lim _{j \rightarrow \infty}\left\|L_{j} f-f\right\|_{2 \pi}=0$, uniformly in $n$, if and only if $s t_{A}-\lim _{j \rightarrow \infty}\left\|L_{j} f_{i}-f_{i}\right\|_{2 \pi}=0$ ( $i=1,2,3)$, uniformly in $n$, where $f_{1}(t)=1, f_{2}(t)=\cos t$, and $f_{3}(t)=\sin t$, for all $t \in \mathscr{R}$.

The following notations are used this paper (see $[5,6])$.

Let $n$ be fixed and sufficiently large. If $y_{i} \in I_{j(i)}$ and $1 \leq$ $i \leq k$, then it is convenient to denote

$$
\begin{aligned}
y_{i}^{\prime} & =x_{j(i)+1}, \\
y_{i}^{\prime \prime} & =x_{j(i)-2}, \\
I_{i}^{\prime} & =\left[y_{i}^{\prime}, y_{i}^{\prime \prime}\right]=I_{j(i)+1} \cup I_{j(i)} \cup I_{j(i)-1} \\
& =\left[x_{j(i)+1}, x_{j(i)}\right] \cup\left[x_{j(i)}, x_{j(i)-1}\right] \cup\left[x_{j(i)-1}, x_{j(i)-2}\right], \\
\rho_{i} & =\left[\frac{y_{i}+y_{i}^{\prime}}{2}, \frac{y_{i}+y_{i}^{\prime \prime}}{2}\right], \quad \text { for } 1 \leq i \leq k, \\
\frac{5}{3} h_{j(i)} & =\frac{5}{3}\left(x_{j(i)-1}-x_{j(i)}\right)<\left(x_{j(i)-2}-x_{j(i)+1}\right)=\left|I_{i}^{\prime}\right| \\
& =2\left|\rho_{i}\right|=\left(y_{i}^{\prime \prime}-y_{i}^{\prime}\right)=\left(x_{j(i)-2}-x_{j(i)+1}\right)<7, \\
h_{j(i)} & =7\left(x_{j(i)-1}-x_{j(i)}\right), \quad 1 \leq i \leq k,
\end{aligned}
$$

and therefore $\left|I_{i}^{\prime}\right| \sim\left|\rho_{i}\right| \sim h_{j(i)}$, for $x \in I_{i}^{\prime}$. Recall that

$$
\operatorname{sgn}(f(x))= \begin{cases}1 ; & \text { if } x \in[a, b] \\ -1 ; & \text { if } x \notin[a, b]\end{cases}
$$

is the sign of $f$ on $[a, b]$.

Now, let us introduce our theorems as follows.

Theorem 3. Let $A=\left(A^{n}\right)_{n \geq 1}$ be a sequence of infinite nonnegative real matrices such that $\sup _{n, k} \sum_{j=1}^{\infty} a_{k j}^{n}<\infty$ and let $\left\{L_{j}\right\}$ be a sequence of positive linear operators mapping $\mathrm{C}_{2 \pi}(\mathscr{R})$ into $\mathrm{C}_{2 \pi}(\mathscr{R})$. Then, for all $f \in \mathrm{C}_{2 \pi}(\mathscr{R})$, we have

$$
\sum_{j=1}^{\infty} a_{k j}^{n}\left\|L_{j} f-f\right\|_{2 \pi} \leq c \omega_{3}^{\phi}\left(f, \frac{\pi}{n},[-\pi, \pi]\right)
$$

uniformly in $n$, if and only if

$$
\sum_{j=1}^{\infty} a_{k j}^{n}\left\|L_{j} f_{\zeta}-f_{\zeta}\right\|_{2 \pi} \leq c \omega_{3}^{\phi}\left(f_{\zeta}, \frac{\pi}{n},[-\pi, \pi]\right), \quad \zeta=1,2,3
$$

uniformly in $n$, where $f_{1}(t)=1, f_{2}(t)=\left(t-y_{i}^{\prime}\right) /\left(y_{i}^{\prime \prime}-y_{i}\right)$, and $f_{3}(t)=\left(t-y_{i}^{\prime \prime}\right) /\left(y_{i}-y_{i}^{\prime}\right)$, for all $t \in \mathscr{R}$. And $c$ the constant does not depend on $j$.

Theorem 4. Let $A=\left(A^{n}\right)_{n \in N}$ be a sequence of nonnegative regular summability matrices and let $\left\{L_{j}\right\}$ be a sequence of positive linear operators mapping $\mathrm{C}_{2 \pi}(\mathscr{R})$ into $\mathrm{C}_{2 \pi}(\mathscr{R})$. Then, if there exists $f \in \mathrm{C}_{2 \pi}(\mathscr{R})$, we have

$$
s t_{A}-\lim _{n}\left\|L_{j} f-f\right\|_{2 \pi} \geq c(j) \omega_{3}^{\phi}\left(f, \frac{\pi}{n},[-\pi, \pi]\right),
$$

uniformly in $n$, if and only if

$$
\begin{aligned}
s t_{A}-\lim _{n}\left\|L_{j} f_{\zeta}-f_{\zeta}\right\|_{2 \pi} & \geq c(j) \omega_{3}^{\phi}\left(f_{\zeta}, \frac{\pi}{n},[-\pi, \pi]\right) \\
\zeta & =1,2,3
\end{aligned}
$$

uniformly in $n$, where $f_{1}(t)=1, f_{2}(t)=\left(t-y_{i}^{\prime}\right) /\left(y_{i}^{\prime \prime}-y_{i}\right)$, and $f_{3}(t)=\left(t-y_{i}^{\prime \prime}\right) /\left(y_{i}-y_{i}^{\prime}\right)$, for all $t \in \mathscr{R}$.

\section{Proofs of Theorems 3 and 4}

Proof of Theorem 3. Since $f_{\zeta}(\zeta=1,2,3)$ belong to $C_{2 \pi}(\mathscr{R})$, implications $(5) \Rightarrow(6)$ are obvious. Now, assume that (6) holds. Let $f \in \mathrm{C}_{2 \pi}(\mathscr{R})$, and, $I$ be a closed subinterval of length $2 \pi$ of $\mathscr{R}$. And let $L_{j}$ be defined by

$$
\begin{gathered}
L_{j}(x)=\frac{x_{n}-y_{i}}{y_{i}^{\prime \prime}-y_{i}^{\prime}}\left(\frac{x-y_{i}^{\prime}}{y_{i}^{\prime \prime}-y i} L_{j}\left(y_{i}^{\prime \prime}\right)-\frac{x-y_{i}^{\prime \prime}}{y_{i}-y_{i}^{\prime}} L_{j}\left(y_{i}^{\prime}\right)\right), \\
y_{i} \in\left[\frac{y_{i}+y_{i}^{\prime}}{2}, \frac{y_{i}+y_{i}^{\prime \prime}}{2}\right] \quad \text { for } i=1,2,3,
\end{gathered}
$$

and also where $L_{j}\left(y_{i}^{\prime}\right)$ and $L_{j}\left(y_{i}^{\prime \prime}\right)$ are chosen so that

$$
\begin{aligned}
L_{j}\left(y_{i}^{\prime}\right) & = \begin{cases}\frac{\mathrm{C}}{\omega_{3}^{\phi}\left(f, n^{-1}\right) \operatorname{sgn}\left(f\left(y_{i}^{\prime}\right)\right)} ; & \text { if }\left|f\left(y_{i}^{\prime}\right)\right| \leq c \omega_{3}^{\phi}\left(f, n^{-1}\right), \\
f\left(y_{i}^{\prime}\right) ; & \text { o.w, }\end{cases} \\
L_{j}\left(y_{i}^{\prime \prime}\right) & = \begin{cases}\frac{\mathrm{C}}{\omega_{3}^{\phi}\left(f, n^{-1}\right) \operatorname{sgn}\left(f\left(y_{i}^{\prime \prime}\right)\right)} ; & \text { if }\left|f\left(y_{i}^{\prime \prime}\right)\right| \leq c \omega_{3}^{\phi}\left(f, n^{-1}\right), \\
f\left(y_{i}^{\prime \prime}\right) ; & \text { o.w. }\end{cases}
\end{aligned}
$$


In [5] Kopotun, we have $|f(x)-L(f ; x)| \leq c \omega_{3}^{\phi}\left(f, n^{-1}\right)$ and $x \in I$, where

$$
\begin{aligned}
L(f ; x) & =L\left(f ; x \mid y_{i}, y_{i}^{\prime}, y_{i}^{\prime \prime}\right) \\
& =\frac{x_{n}-y_{i}}{y_{i}^{\prime \prime}-y_{i}^{\prime}}\left(\frac{x-y_{i}^{\prime}}{y_{i}^{\prime \prime}-y_{i}} L_{j}\left(y_{i}^{\prime \prime}\right)-\frac{x-y_{i}^{\prime \prime}}{y_{i}-y_{i}^{\prime}} L_{j}\left(y_{i}^{\prime}\right)\right)
\end{aligned}
$$

is the Lagrange polynomial of degree $\leq 2$, which interpolates $f$ at $y_{i}, y_{i}^{\prime}$, and $y_{i}^{\prime \prime}$. Inequality (11) is an analog of Whitney's inequality for Ditzian-Totik moduli. Using (11) and the above presentations of $L_{j}$ and $L(f ; x)$, we write, for $x \in I$,

$$
\begin{aligned}
& \left|L_{j}(f ; x)-f(x)\right| \\
& \quad \leq\left|L_{j}(f ; x)-L(f ; x)\right|+|L(f ; x)-f(x)| \\
& \leq\left|\frac{\left(x_{n}-y_{i}\right)\left(x-y_{i}^{\prime}\right)}{\left(y_{i}^{\prime \prime}-y_{i}^{\prime}\right)\left(y_{i}^{\prime \prime}-y_{i}\right)}\right|\left|L_{j}\left(y_{i}^{\prime \prime}\right)-f\left(y_{i}^{\prime \prime}\right)\right| \\
& \quad+\left|\frac{\left(x_{n}-y_{i}\right)\left(x-y_{i}^{\prime \prime}\right)}{\left(y_{i}^{\prime \prime}-y_{i}^{\prime}\right)\left(y_{i}^{\prime \prime}-y_{i}\right)}\right| \\
& \quad \times\left|L_{j}\left(y_{i}^{\prime}\right)-f\left(y_{i}^{\prime}\right)\right|+|L(f ; x)-f(x)| .
\end{aligned}
$$

Taking supremum over $x$ and $=1 /\left(\omega_{3}^{\phi}\left(f, n^{-1}\right) \operatorname{sgn}(f)\right)$, we obtain

$$
\begin{aligned}
&\left\|L_{j}(f ; x)-f(x)\right\|_{2 \pi} \\
& \leq\left\|L_{j}(f ; x)-L(f ; x)\right\|_{2 \pi}+\|L(f ; x)-f(x)\|_{2 \pi} \\
& \leq\left|\frac{\left(x_{n}-y_{i}\right)\left(x-y_{i}^{\prime}\right)}{\left(y_{i}^{\prime \prime}-y_{i}^{\prime}\right)\left(y_{i}^{\prime \prime}-y_{i}\right)}\right|\left\|L_{j}\left(y_{i}^{\prime \prime}\right)-f\left(y_{i}^{\prime \prime}\right)\right\|_{2 \pi} \\
&+\left|\frac{\left(x_{n}-y_{i}\right)\left(x-y_{i}^{\prime \prime}\right)}{\left(y_{i}^{\prime \prime}-y_{i}^{\prime}\right)\left(y_{i}^{\prime \prime}-y_{i}\right)}\right|\left\|L_{j}\left(y_{i}^{\prime}\right)-f\left(y_{i}^{\prime}\right)\right\|_{2 \pi} \\
&+\|L(f ; x)-f(x)\|_{2 \pi} \\
& \leq c\left(K, y_{i}^{\prime \prime}\right)+c\left(K, y_{i}^{\prime}\right)+c \omega_{3}^{\phi}\left(f, n^{-1},[-1,1]\right) .
\end{aligned}
$$

Suppose $B>0$, let us write sets as follows:

$$
\begin{aligned}
\mathcal{\vartheta}=\{ & j:\left\|L_{j}(1 ; x)-1\right\|_{2 \pi} \\
& +\left\|L_{j}\left(\frac{t-y_{i}^{\prime}}{y_{i}^{\prime \prime}-y_{i}} ; x\right)-\frac{x-y_{i}^{\prime}}{y_{i}^{\prime \prime}-y_{i}}\right\|_{2 \pi} \\
& \left.+\left\|L_{j}\left(\frac{t-y_{i}^{\prime \prime}}{y_{i}-y_{i}^{\prime}} ; x\right)-\frac{x-y_{i}^{\prime \prime}}{y_{i}-y_{i}^{\prime}}\right\|_{2 \pi} \geq K B\right\},
\end{aligned}
$$

$$
\begin{aligned}
& \vartheta_{1}=\left\{j:\left\|L_{j}(1 ; x)-1\right\|_{2 \pi} \geq K B\right\}, \\
& \vartheta_{2}=\left\{j:\left\|L_{j}\left(\frac{t-y_{i}^{\prime}}{y_{i}^{\prime \prime}-y_{i}} ; x\right)-\frac{x-y_{i}^{\prime}}{y_{i}^{\prime \prime}-y_{i}}\right\|_{2 \pi} \geq K B\right\}, \\
& \vartheta_{3}=\left\{j:\left\|L_{j}\left(\frac{t-y_{i}^{\prime \prime}}{y_{i}-y_{i}^{\prime}} ; x\right)-\frac{x-y_{i}^{\prime \prime}}{y_{i}-y_{i}^{\prime}}\right\|_{2 \pi} \geq K B\right\} .
\end{aligned}
$$

Consequently, we get $\vartheta \subset \vartheta_{1} \cup \vartheta_{2} \cup \vartheta_{3}$ and $\sum_{j \in \vartheta} a_{k j}^{n} \geq$ $\sum_{j \in \vartheta_{1}} a_{k j}^{n} \geq \sum_{j \in \vartheta_{2}} a_{k j}^{n} \geq \sum_{j \in \vartheta_{3}} a_{k j}^{n}$ implies

$$
\sum_{j=1}^{\infty} a_{k j}^{n}\left\|L_{j} f-f\right\|_{2 \pi} \leq c \omega_{3}^{\phi}\left(f, \frac{\pi}{n},[-\pi, \pi]\right) .
$$

Proof of Theorem 4. Since $f_{\zeta}(\zeta=1,2,3)$ belong to $C_{2 \pi}(\mathscr{R})$, implications $(8) \Rightarrow(7)$ are obvious. Assume that the condition (7) is satisfied. Let $f \in \mathrm{C}_{2 \pi}(\mathscr{R})$ and $I$ be a closed subinterval of length $2 \pi$ of $\mathscr{R}$; we have

$$
s t_{A}-\lim _{n}\left\|L_{j} f-f\right\|_{2 \pi} \geq c(j) \omega_{3}^{\phi}\left(f, \frac{\pi}{n},[-\pi, \pi]\right) .
$$

Now, given $K(j)>0$, choose $B>0$, where $B=$ $\sup \{|f(x)|: x \in I\}$ implied $K<B$, and define the following set:

$$
\begin{aligned}
\mathcal{\vartheta}=\left\{j:\left\|L_{j}(1 ; x)-1\right\|_{2 \pi}\right. \\
+\left\|L_{j}\left(\frac{t-y_{i}^{\prime}}{y_{i}^{\prime \prime}-y_{i}} ; x\right)-\frac{x-y_{i}^{\prime}}{y_{i}^{\prime \prime}-y_{i}}\right\|_{2 \pi} \\
\left.+\left\|L_{j}\left(\frac{t-y_{i}^{\prime \prime}}{y_{i}-y_{i}^{\prime}} ; x\right)-\frac{x-y_{i}^{\prime \prime}}{y_{i}-y_{i}^{\prime}}\right\|_{2 \pi} \geq K B\right\} .
\end{aligned}
$$

Thus,

$$
\begin{aligned}
s t_{A} & -\lim _{n}\left\|L_{j} p_{3}(t)-p_{3}(x)\right\|_{2 \pi} \\
& =s t_{A}-\lim _{n}\left\|L_{j}\left(\frac{t-y_{i}^{\prime \prime}}{y_{i}-y_{i}^{\prime}} ; x\right)-\frac{x-y_{i}^{\prime \prime}}{y_{i}-y_{i}^{\prime}}\right\|_{2 \pi} \\
& \geq\left(K-K_{\circ}\right) \grave{B},
\end{aligned}
$$

where $p_{3}(x)=\left(x-y_{i}^{\prime \prime}\right) /\left(y_{i}-y_{i}^{\prime}\right) \in \mathrm{C}_{2 \pi}(\mathscr{R})$ polynomial and $x \in \mathscr{R}$. Since $x$ is $A$-statistically convergent, we can easily show that $\mathcal{Y} \supset \vartheta_{1} \supset \vartheta_{2} \supset \vartheta_{3}$ implies $\sum_{j \in \vartheta} a_{k j}^{n} \geq \sum_{j \in \vartheta_{1}} a_{k j}^{n} \geq$ $\sum_{j \in \vartheta_{2}} a_{k j}^{n} \geq \sum_{j \in \vartheta_{3}} a_{k j}^{n}$.

Now, let $\grave{B}=\omega_{3}^{\phi}\left(f_{\zeta}, \pi / n,[-\pi, \pi]\right)$, and using (7) implies

$$
s t_{A}-\lim _{n}\left\|L_{j} p_{3}(t)-p_{3}(x)\right\|_{2 \pi} \geq c(j) \omega_{3}^{\phi}\left(p_{3}, \frac{\pi}{n},[-\pi, \pi]\right) .
$$

This is a complete proof. 


\section{Application to Functional Approximation}

In this section we give some applications which satisfy our theorems, but it's not the classical Korovkin theorem. It has been treated with the Weierstrass second approximation theorem via $A$-statistical convergence (see [6-8]). If $f \in \mathrm{C}_{2 \pi}(\mathscr{R})$, then there is a sequence of polynomials and $A$-statistically uniformly convergent to $f$ on $[-\pi, \pi]$ (not uniformly convergent). Observe that Fejer operators may be written in the form of

$$
F_{n}(f ; x)=\frac{a_{\circ}}{2}+\sum_{k=1}^{n} \frac{n-k}{n}\left(a_{k} \frac{k x-y_{i}^{\prime}}{y_{i}^{\prime \prime}-y_{i}}+b_{k} \frac{k x-y_{i}^{\prime \prime}}{y_{i}-y_{i}^{\prime}}\right) .
$$

We now consider the linear operator $T_{n}$ defined by

$$
T_{n}(f ; x)=\frac{a_{\circ}}{2}+\sum_{k=1}^{n} \xi_{k}^{(n)}\left(a_{k} \frac{k x-y_{i}^{\prime}}{y_{i}^{\prime \prime}-y_{i}}+b_{k} \frac{k x-y_{i}^{\prime \prime}}{y_{i}-y_{i}^{\prime}}\right)
$$

where $\left\{\xi_{k}^{(n)}\right\}(n=1,2, \ldots ; k=1,2, \ldots, n)$ is a matrix of real numbers and also $a_{k}$ and $b_{k}$ are Fourier coefficients. Now, let $A=\left(a_{n k}\right)$ be a nonnegative regular summability matrice. Assume that the following statements are satisfied:

(i) $s t_{A}-\lim _{n} \xi_{1}^{(n)}=1$;

(ii) $(1 / 2)+\sum_{k=1}^{n} \xi_{k}^{(n)}\left(t-y_{i}^{\prime}\right) /\left(y_{i}^{\prime \prime}-y_{i}\right) \geq c(n) \omega_{3}^{\phi}(f, \pi / n$, $[-\pi, \pi])$. We get

$$
\begin{aligned}
& s t_{A}-\lim _{n}\left\|T_{n}(f ; x)-f(x)\right\|_{2 \pi} \\
& \quad \geq c(n) \omega_{3}^{\phi}\left(f, \frac{\pi}{n},[-\pi, \pi]\right), \quad \forall f \in \mathrm{C}_{2 \pi}(\mathscr{R}),
\end{aligned}
$$

where $\left\{T_{n}\right\}$ is the sequence of linear operators given by $(21)$.

In [9], Sakaoğlu and Ünver proved the following theorem by using $L_{P}[a, b ; c, d]$ and denoted the space of all functions $f$ defined on $[a, b] \times[c, d]$, for which $\int_{c}^{d} \int_{a}^{b}|f(x, y)|^{P} d x d y<$ $\infty, 1 \leq P<\infty$. In this case, the $L_{P}$ norm of a function $f$ in $L_{P}[a, b ; c, d]$, denoted by $\|f\|_{P}$, is given by $\|f\|_{P}=$ $\left(\int_{c}^{d} \int_{a}^{b}|f(x, y)|^{P} d x d y\right)^{1 / P}$.

Theorem 5 (see [9]). Let $A=\left(a_{j n}\right)$ be a nonnegative regular summability matrix and let $\left\{T_{n}\right\}$ be an A-statistically uniformly bounded sequence of positive linear operators from $L_{P}[a, b ; c, d]$ into $L_{P}[a, b ; c, d]$ and $1 \leq P<\infty$. Then, for any function $f \in L_{P}[a, b ; c, d], s t_{A}-\lim _{n}\left\|T_{n}(f ; x, y)-f(x, y)\right\|_{P}=$ 0 if and only if $s t_{A}-\lim _{n}\left\|T_{n}\left(f_{i} ; x, y\right)-f_{i}(x, y)\right\|_{P}=0, i=$ $1,2,3,4$ where $f_{1}(t, v)=1, f_{2}(t, v)=t, f_{3}(t, v)=v$, and $f_{4}(t, v)=t^{2}+v^{2}$.

The theory of the Lebesgue integral can be developed in several distinct ways (see $[10,11])$. Only one of these methods will be discussed here.

Now, let us introduce our definition as follows.

Definition 6 (Lebesgue-Stieltjes integral-i). Let $S$ be measurable set, $f: S \rightarrow R$ be a bounded function, and $g_{i}: S \rightarrow$
$R$ be nondecreasing function for $i \in I$. For $\mathscr{P}$ Lebesgue partition of $S$, put $\underline{\operatorname{LS}}(f, \mathscr{P}, \underline{g})=\sum_{j=1}^{n} \prod_{i \in I} m_{j} g_{i}\left(\mu\left(S_{j}\right)\right)$ and $\overline{\mathrm{LS}}(f, \mathscr{P}, g)=\sum_{j=1}^{n} \prod_{i \in I} \bar{M}_{j} g_{i}\left(\mu\left(S_{j}\right)\right)$ such that $\mu$ measurable function of $S ; m_{j}=\inf \left\{f(x): x \in S_{j}\right\}$, $M_{j}=\sup \left\{f(x): x \in S_{j}\right\}$, and $g=g_{1}, g_{2}, \ldots$ Also, $g_{i}\left(x_{j}\right)-g_{i}\left(x_{j-1}\right)>0, \underline{\mathrm{LS}}(f, \mathscr{P}, \underline{g}) \leq \overline{\mathrm{LS}}(f, \mathscr{P}, \underline{g})$, $\prod_{i \in I} \int_{i} f \underline{\mathrm{d} g}=\sup \{\underline{\mathrm{LS}}(f, \underline{g})\}$, and $\prod_{i \in I} \overline{\int_{i}} f \underline{\mathrm{d} g}=\inf \{\overline{\mathrm{LS}}(f, \underline{g})\}$, where $\underline{\underline{L S}}(f, g)=\{\underline{\mathrm{LS}}(f, \mathscr{P}, g): \overline{\mathscr{P}}$ part of set $S\}$ and $\overline{\mathrm{LS}}(f, \underline{g})=\{\overline{\mathrm{LS}}(f, \mathscr{P}, \underline{g}): \mathscr{P}$ part of set $S\}$. If $\prod_{i \in I} \int_{i} f \underline{\mathrm{d} g}=$ $\prod_{i \in I} \overline{\int_{i}} f \mathrm{~d} g$, where $\mathrm{d} g=\mathrm{d} g_{1} \times \mathrm{d} g_{2} \times \ldots \times \mathrm{d} g_{n} \ldots$. Then $f$ is integral $\int_{i}$ according to $g_{i}$ for $i \in I$.

Now, we can provide our theorem as follows as a case which is an illustrative application of approximation theory in functional analysis using functional supremum to limit convergence that acts as support and reinforcement of the concept of Riesz's representation.

Theorem 7. If a sequence $G_{n}(f)$ is positive linear functional and bounded on $C(S), f$ is bounded measurable function to $S$. Then, there exists nondecreasing function to $S$ such that $s t_{A}-$ $\lim _{\mu(S) \rightarrow 0}\left(\sup _{n} G_{n}(f)-f\right)=0$.

Proof. Assume that functional supremum $G_{n}$ is as follows:

$$
\sup _{n} G_{n}(f)=\sup _{n} \prod_{i \in I} \int_{i} f \underline{\mathrm{d} \varphi_{t, n}}
$$

where $\varphi_{t, n}(x)=(1-n(x-t)) /\left(y_{i}^{\prime \prime}-y_{i}^{\prime}\right)$ converges to $r \in R$; that is, let $\mathscr{P}=\left\{\mathrm{S}_{\ell}\right\}_{\ell=0}^{m}$ be Lebesgue partition such that

$$
\begin{gathered}
\sup \left\{\mu\left(\mathrm{S}_{\ell}\right): \ell=0, \ldots, m\right\}<\frac{1}{2} \delta(\varepsilon), \\
\frac{1}{2}<\inf \left\{\mu\left(\mathrm{S}_{\ell}\right): \ell=0, \ldots, m\right\}, \\
\varphi_{i t, n}\left(\mu\left(\mathrm{S}_{\ell}\right)\right) \leq G\left(\pi_{t, n}\left(\mu\left(\mathrm{S}_{\ell}\right)\right)\right) \leq \varphi_{i t, n}\left(\mu\left(\mathrm{S}_{\ell}\right)\right)+\frac{\varepsilon}{m}\|f\|,
\end{gathered}
$$

where $\pi_{t, n}(x)=\varphi_{t, n}(x)\left(y_{i}^{\prime \prime}-y_{i}^{\prime}\right)$.

Since $G$ positive linear functional and bounded on $C(S)$, then

$$
\begin{aligned}
& \left|G(f)-G\left(f\left(t_{1}\right) \pi_{t_{1}, n}+\sum_{\mathscr{L}=2}^{m} f\left(t_{\mathscr{L}}\right) \pi_{t_{\mathscr{L}, n}}\left(\mu\left(\mathrm{S}_{\ell}\right)\right)\right)\right| \\
& \quad<\frac{\varepsilon}{m}\|f\|,
\end{aligned}
$$


also, respect between sum $\operatorname{LS}\left(f, \mathscr{P}, \underline{\varphi_{t, n}}\right)$ and LebesgueStieltjes integral- $i$ are $2 \varepsilon$, we have

$$
\begin{aligned}
& \left|\prod_{i \in I} \int_{i} f \underline{\mathrm{d} \varphi_{t, n}}-G(f)\right| \\
& \leq \mid \prod_{i \in I} \int_{i} f \underline{\mathrm{d} \varphi_{t, n}} \\
& \quad-G\left(f\left(t_{1}\right) \pi_{t_{1}, n}+\sum_{\mathscr{L}=2}^{m} f\left(t_{\mathscr{L}}\right) \pi_{t_{\mathscr{L}}, n}\left(\mu\left(\mathrm{S}_{\ell}\right)\right)\right) \mid \\
& \quad+\left|G(f)-G\left(f\left(t_{1}\right) \pi_{t_{1}, n}+\sum_{\mathscr{L}=2}^{m} f\left(t_{\mathscr{L}}\right) \pi_{t_{\mathscr{L}, n}}\left(\mu\left(\mathrm{S}_{\ell}\right)\right)\right)\right| \\
& <\frac{\grave{\varepsilon}}{m}
\end{aligned}
$$

as $m \rightarrow \infty$; hence $G$ satisfies Lebesgue-Stieltjes integral- $i$ of $f$.

Now, since $G_{n}(f)$ is functional supremum and satisfies Lebesgue-Stieltjes integral- $i$, and us Definition 6, we have

$$
\begin{aligned}
& \sup _{n} G_{n}(f)-f \\
& =\sup _{n} \prod_{i \in I} \int_{i} f \underline{\mathrm{d} \varphi_{t, n}-f} \\
& =\sup _{n}\left(\sup \sum_{j=1}^{n} \prod_{i \in I} m_{j} \varphi_{i t, n}\left(\mu\left(S_{j}\right)\right)\right)-f \\
& =\sup _{n}\left(\sup \sum_{j=1}^{n} \prod_{i \in I} \inf _{j} f(x) \varphi_{i t, n}\left(\mu\left(S_{j}\right)\right)\right)-f \\
& =\sup _{n}\left(\inf \sum_{j=1}^{n} \prod_{i \in I} \sup _{j} f(x) \varphi_{i t, n}\left(\mu\left(S_{j}\right)\right)\right)-f \\
& =\sup _{n}\left(\inf \sum_{j=1}^{n} \sup _{j} f(x) \prod_{i \in I} \varphi_{i t, n}\left(\mu\left(S_{j}\right)\right)\right)-f \\
& =\sup _{n}\left(\inf \sum_{j=1}^{n} \sup _{j} f(x)\right. \\
& \quad \times\left(\left[\left(\frac{1-n\left(\mu\left(S_{1}\right)-t\right)}{y_{i}^{\prime \prime}-y_{i}^{\prime}}\right)\right.\right. \\
& =\sup _{n}\left(\operatorname { i n f } \left(\sup _{1} f(x)\right.\right. \\
& \left.\quad\left(\frac{1-n\left(\mu\left(S_{2}\right)-t\right)}{y_{i}^{\prime \prime}-y_{i}^{\prime}}\right) \ldots\right]
\end{aligned}
$$

$$
\begin{aligned}
& \times\left[\left(\frac{1-n\left(\mu\left(S_{1}\right)-t\right)}{y_{i}^{\prime \prime}-y_{i}^{\prime}}\right)\right. \\
& \left.\cdot\left(\frac{1-n\left(\mu\left(S_{2}\right)-t\right)}{y_{i}^{\prime \prime}-y_{i}^{\prime}}\right) \cdots\right] \\
& +\sup _{2} f(x)\left[\left(\frac{1-n\left(\mu\left(S_{1}\right)-t\right)}{y_{i}^{\prime \prime}-y_{i}^{\prime}}\right)\right. \\
& \left.+\cdot\left(\frac{1-n\left(\mu\left(S_{2}\right)-t\right)}{y_{i}^{\prime \prime}-y_{i}^{\prime}}\right) \cdots\right] \\
& \times\left[\left(\frac{1-n\left(\mu\left(S_{1}\right)-t\right)}{y_{i}^{\prime \prime}-y_{i}^{\prime}}\right)\right. \\
& \left.\left.\left.\quad \cdot\left(\frac{1-n\left(\mu\left(S_{2}\right)-t\right)}{y_{i}^{\prime \prime}-y_{i}^{\prime}}\right) \cdots\right]\right)\right)-f .
\end{aligned}
$$

Note that effect sum on measurable function $\mu\left(S_{j}\right)$ by using Lebesgue partition

$$
\lim _{\mu(S) \rightarrow 0} \prod_{i \in I} \int_{i} f \underline{\mathrm{d} \varphi_{t, n}}=f ;
$$

let $n \in N$, choose $\mathscr{K}_{n}>0$, and define the following sets:

$$
\begin{aligned}
\mathbb{L} & =\left\{\mu(S):\left(\sup _{n} G_{n}(f)-f\right) \geq \mathscr{K}_{n}\right\}, \\
\mathbb{L}_{1} & =\left\{\mu(S):\left(\sup _{n} G_{n}\left(f_{1}\right)-f_{1}\right) \geq \frac{\mathscr{K}_{n}}{3}\right\}, \\
\mathbb{L}_{2} & =\left\{\mu(S):\left(\sup _{n} G_{n}\left(f_{2}\right)-f_{2}\right) \geq \frac{\mathscr{K}_{n}}{3}\right\}, \\
\mathbb{L}_{3} & =\left\{\mu(S):\left(\sup _{n} G_{n}\left(f_{3}\right)-f_{3}\right) \geq \frac{\mathscr{K}_{n}}{3}\right\} ;
\end{aligned}
$$

Then $\mathbb{L} \subset \mathbb{L}_{1} \cup \mathbb{L}_{2} \cup \mathbb{L}_{3}$, which gives

$$
\sum_{\mu(\mathrm{S}) \subset \mathbb{L}} a_{k j}^{n} \leq \sum_{\mu(\mathrm{S}) \subset \mathbb{L}_{1}} a_{k j}^{n} \cup \sum_{\mu(\mathrm{S}) \subset \mathbb{L}_{2}} a_{k j}^{n} \cup \sum_{\mu(\mathrm{S}) \subset \mathbb{L}_{3}} a_{k j}^{n} ;
$$

we obtain that $s t_{A}-\lim _{\mu(S) \rightarrow 0} \sum_{\mu(S) \subset \mathbb{L}} a_{k j}^{n}=0$ implies $s t_{A}-$ $\lim _{\mu(S) \rightarrow 0}\left(\sup _{n} G_{n}(f)-f\right)=0$.

Now, in this paper we have proved Riesz's representation theory with Lebesgue-Stieltjes integral-i, by using Korovkin type approximation which is one of the threads in the development of Riesz's theorem to support the definition of Lebesgue integral, Rudin [10]. This integration toxicity ratio for the world on behalf of the French Lebesgue, who came in his thesis for a doctorate in 1902.

\section{Conflict of Interests}

The author declares that there is no conflict of interests regarding the publication of this paper. 


\section{Acknowledgments}

The author is grateful for hospitality at the University of Kufa. He thanks his fellows for the fruitful discussions while preparing this paper. He was partially supported by University of Al-Muthanna.

\section{References}

[1] O. Duman, "Statistical approximation for periodic functions," Demonstratio Mathematica, vol. 36, no. 4, pp. 873-878, 2003.

[2] C. Radu, "A-summability and approximation of continuous periodic functions," Studia Universitatis, Babeş-Bolyai: Mathematica, vol. 52, no. 4, pp. 155-161, 2007.

[3] G. A. Dzyubenko and J. Gilewicz, "Copositive approximation of periodic functions," Acta Mathematica Hungarica, vol. 120, no. 4, pp. 301-314, 2008.

[4] Z. Ditzian and V. Totik, Moduli of Smoothness, Springer, Berlin, Germany, 1987.

[5] K. Kopotun, "On copositive approximation by algebraic polynomials," Analysis Mathematica, vol. 21, no. 4, pp. 269-283, 1995.

[6] I. A. Shevchuk, Approximation by Polynomials and Traces of the Functions Continuous on an Interval, Naukova Dumka, Kiev, Ukraine, 1992 (Russian).

[7] P. P. Korovkin, Linear Operators and Approximation Theory, Gordon and Breach, Delhi, India, 1960.

[8] R. J. Serfling, Approximation Theorems of Mathematical Statistics, John Wiley \& Sons, 1980.

[9] I. Sakaoğlu and M. Ünver, "Statistical approximation for multivariable integrable functions," Miskolc Mathematical Notes, vol. 13, no. 2, pp. 485-491, 2012.

[10] W. Rudin, Principles of Mathematical Analysis, McGraw-Hill, 3rd edition, 1976.

[11] R. G. Bartle, The Elements of Real Analysis, John Wiley \& Sons, 3rd edition, 1976. 


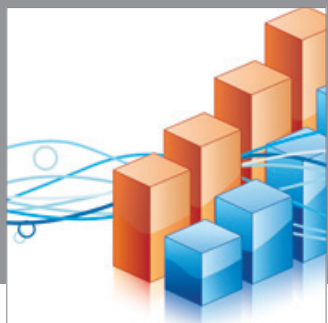

Advances in

Operations Research

mansans

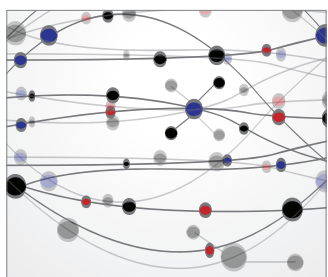

The Scientific World Journal
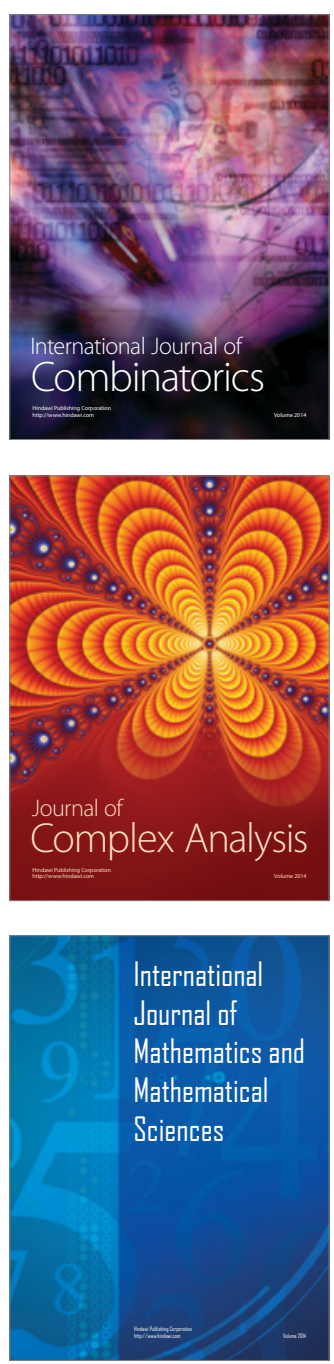
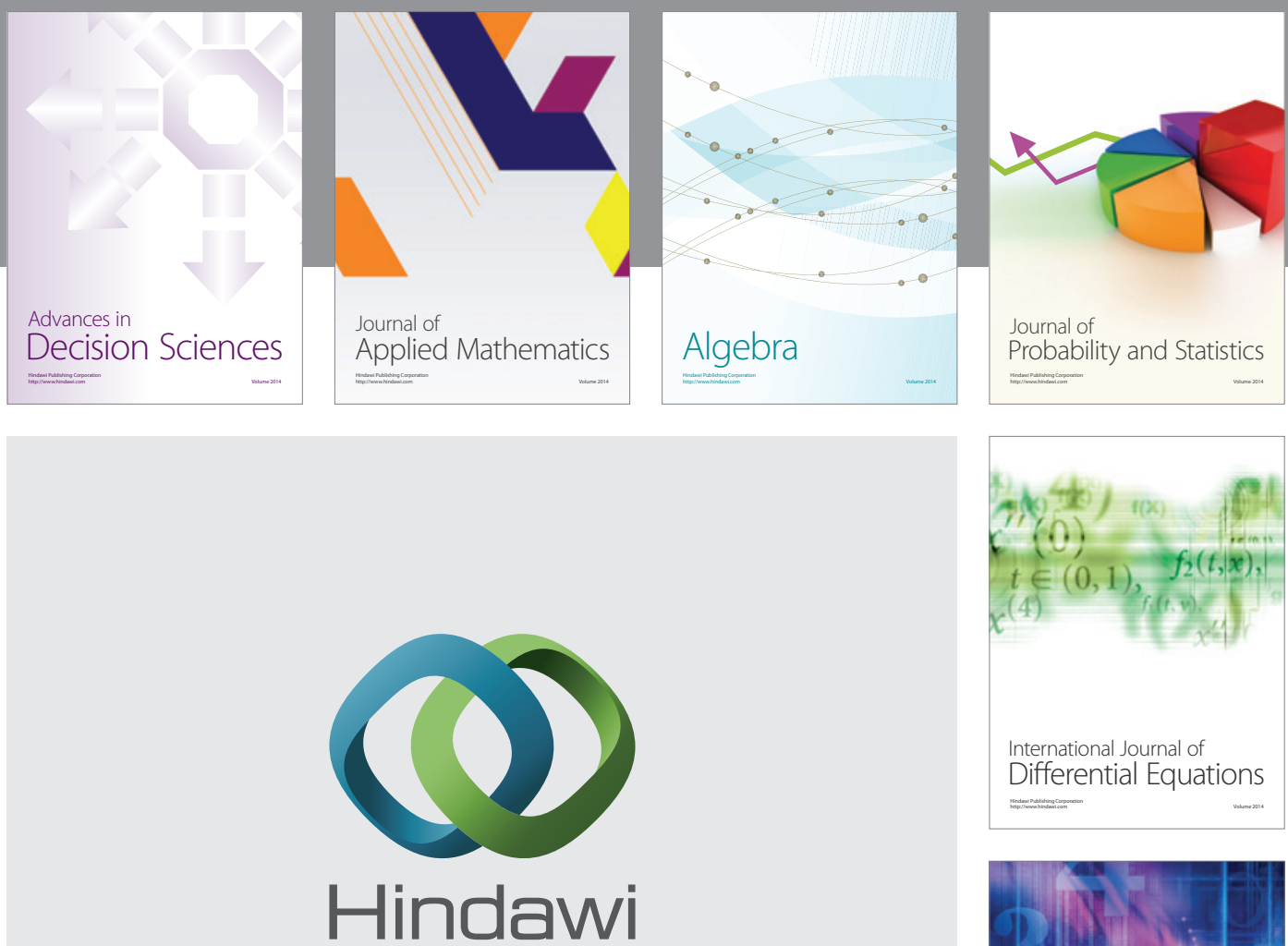

Submit your manuscripts at http://www.hindawi.com
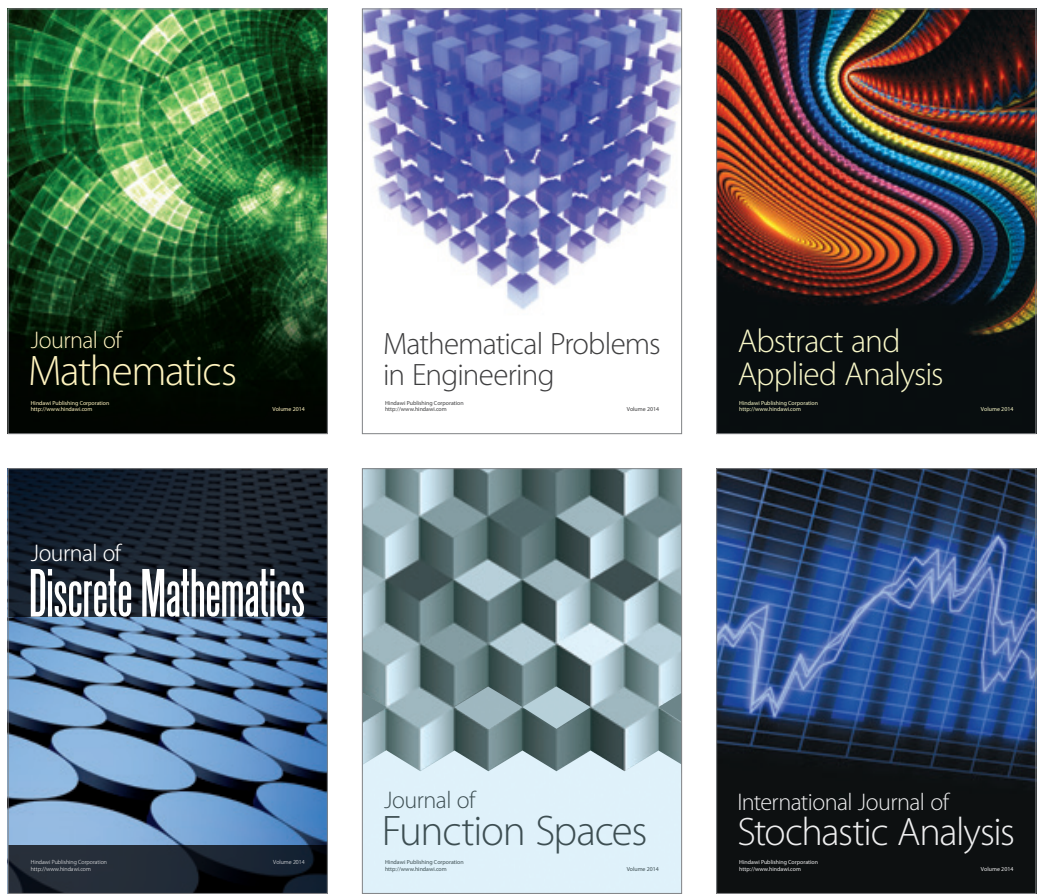

Journal of

Function Spaces

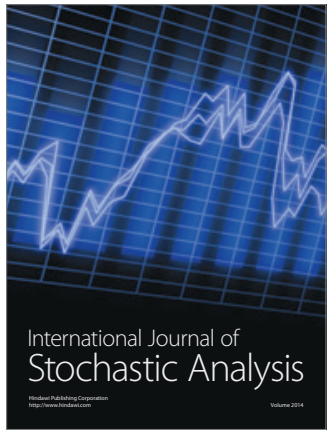

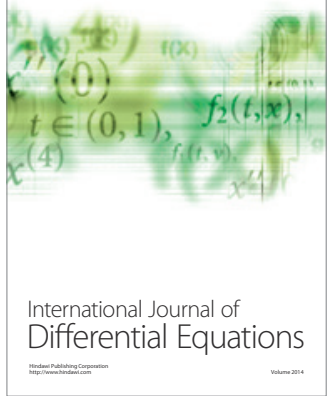
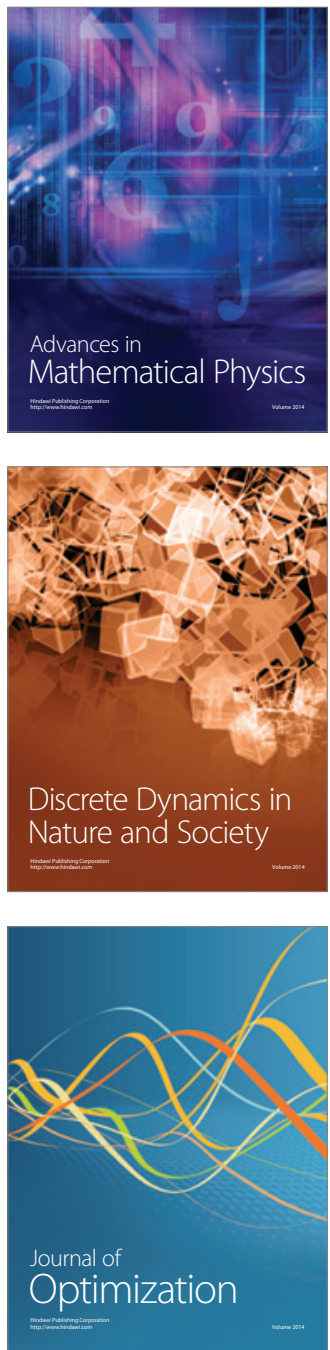